\title{
Stabilizing Parametric Region of Multiloop PID Controllers for Multivariable Systems Based on Equivalent Transfer Function
}

\author{
Xiaoli Luan, ${ }^{1}$ Qiang Chen, ${ }^{1}$ Pedro Albertos, ${ }^{2}$ and Fei Liu ${ }^{1}$ \\ ${ }^{1}$ Key Laboratory of Advanced Process Control for Light Industry, Ministry of Education and the Institute of Automation, \\ Jiangnan University, Wuxi 214122, China \\ ${ }^{2}$ Department of System Engineering and Automatica, Polytechnic University of Valencia, 46022 Valencia, Spain
}

Correspondence should be addressed to Pedro Albertos; pedro@aii.upv.es

Received 9 March 2016; Accepted 3 May 2016

Academic Editor: Stefan Balint

Copyright ( 92016 Xiaoli Luan et al. This is an open access article distributed under the Creative Commons Attribution License, which permits unrestricted use, distribution, and reproduction in any medium, provided the original work is properly cited.

\begin{abstract}
The aim of this paper is to determine the stabilizing PID parametric region for multivariable systems. Firstly, a general equivalent transfer function parameterization method is proposed to construct the multiloop equivalent process for multivariable systems. Then, based on the equivalent single loops, a model-based method is presented to derive the stabilizing PID parametric region by using the generalized Hermite-Biehler theorem. By sweeping over the entire ranges of feasible proportional gains and determining the stabilizing regions in the space of integral and derivative gains, the complete set of stabilizing PID controllers can be determined. The robustness of the design procedure against the approximation in getting the SISO plants is analyzed. Finally, simulation of a practical model is carried out to illustrate the effectiveness of the proposed technique.
\end{abstract}

\section{Introduction}

Despite the rapid development of advanced process control techniques, multiloop proportional integral derivative (PID) control is often used to control multi-input-multi-output (MIMO) systems [1-3]. The main reason for such popularity is its simple control structure and the easiness to handle loop failures. Compared with single-loop controller design, the tuning of multiloop controllers to meet performance requirements is much more difficult because of the interactions among the loops. Much attention has been devoted to achieve good performance in view of the limitation imposed by process interactions, such as the commonly used independent design method [4-6].

Although great progress on multiloop PID control has been achieved in the last decades, some fundamental issues remain to be addressed for better understanding and more effective applications of multiloop PID control to MIMO multidelayed plants. Since the essential requirement imposed on the PID controller is to ascertain the stability of the resulting closed-loop system, the very first task when using PID control is to get a stabilizing PID controller for a given process with time delay [7]. This problem is of great importance, in both theoretical and practical aspects, and it is also related to the problems of stability or robustness. Such a stabilizing set can not only be useful to design PID controllers satisfying defined performance requirements, but also avoid the timeconsuming stability analysis during the controller tuning stage.

Therefore, approaches to design the stabilizing PID controllers have attracted much attention in the process control field over the past decade. In particular, first-order plus dead time (FOPDT) processes [8-12] have been studied and it has been also shown that this kind of stability analysis is also useful in the design context [13-15]. A solution for linear time-invariant systems using low-order controller has been proposed in [16]. Explicit solutions have been also introduced for the case of integral processes [17-19]. Unfortunately, the aforementioned approaches are still only limited to the single-input-single-output (SISO) systems. To the best of our knowledge, no result is available to find the stabilizing PID parametric region for MIMO systems. This fact motivates the present paper to provide a complete solution to the problem of characterizing the set of all multiloop PID parameters stabilizing multivariable systems with time delays. 


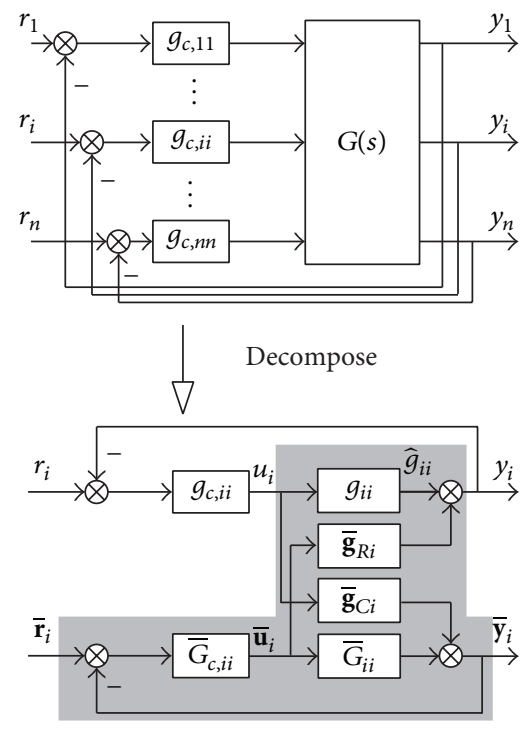

Figure 1: Multivariable control system and each equivalent single loop.

In this paper, the procedures for determining all stabilizing parameters under multiloop PID control structure for multivariable systems are provided. The first part is to decompose the multivariable system into an assembly of SISO models. Then, a model-based method is presented to determine the range of stabilizing proportional gain for SISO plants. Finally, a region with known shape and boundaries is used to show the stabilizing integral and derivative gains. The results of the stabilizing multiloop PID parameter range are obtained individually without interacting with each other. The robustness of the design procedure against the approximation in getting the SISO plants is analyzed. Simulation given in the last section illustrates the effectiveness of the stabilizing PID parametric region found by the proposed technique.

\section{Equivalent Single Loops}

A regular $n$ multivariable system with diagonal pairing, which is controlled with a multiloop controller, is drafted in Figure 1. By considering the interactions among the loops, the multivariable system is decomposed into a set of equivalent single loops. Each one of them is represented by its so-called equivalent transfer function (ETF) [20-22]. The ETF $\hat{g}_{i i}$ has been shown as shaded area in Figure 1. Let us denote by $\mathbf{r}, \mathbf{u}$, and $\mathbf{y}$ the vector of reference inputs, manipulated variables, and system outputs, respectively, and by $G_{c}(s)$ the diagonal matrix of one-by-one controllers.

Notations in Figure 1 are as follows: $\overline{\mathbf{r}}_{i}, \overline{\mathbf{u}}_{i}$, and $\overline{\mathbf{y}}_{i}(i=$ $1,2, \ldots, n)$ are the $\mathbf{r}, \mathbf{u}$, and $\mathbf{y}$ vectors, where $r_{i}, u_{i}$, and $y_{i}$ have been discarded, respectively. $\bar{G}_{c, i i}$ represents the diagonal controller where the $i$ th row and $i$ th column elements of $G_{c}(s)$ have been removed. Similarly, $\bar{G}_{i i}$ represents the transfer function matrix in which the $i$ th row and $i$ th column elements of $G(s)$ are removed. $\overline{\mathbf{g}}_{R i}$ is the row vector of the $i$ th row elements of $G(s)$ when the $i$ th column elements were dropped from $G(s)$, and $\overline{\mathbf{g}}_{C i}$ is the column vector of the $i$ th column elements of $G(s)$ when the $i$ th row elements were dropped from $G(s)$.

When the overall system is closed, there are interactions among control loops as a result of the existence of offdiagonal elements in the transfer matrix. Considering the interactions from other loops, the ETFs can be compactly expressed as follows:

$$
\widehat{g}_{i i}=g_{i i}-\overline{\mathbf{g}}_{R i} \bar{G}_{c, i i}\left(I+\bar{G}_{i i} \bar{G}_{c, i i}\right)^{-1} \overline{\mathbf{g}}_{C i}
$$

where $\hat{g}_{i i}(s)$ is denoted as the ETF.

Assumption 1. Under "perfect" control of the remaining loops, that is, for $y_{j}(s) \cong r_{j}(s), j \neq i$, the following approximation is valid:

$$
\widehat{g}_{i i}=g_{i i}-\overline{\mathbf{g}}_{R i} \overline{\mathbf{g}}_{C i} .
$$

This simplification is similar to the one used for the wellknown RGA (relative gain array) used in evaluating the variables coupling. Since the controllers to be designed include integral actions to avoid offset, a perfect control approximation can be considered at the frequencies lower than the crossover frequency [4]. This can be expressed as

$$
\bar{G}_{c, i i}\left(I+\bar{G}_{i i} \bar{G}_{c, i i}\right)^{-1} \cong I .
$$

Therefore, according to the appendix of [4], (1a) is reasonably simplified as (1b). It can be expressed as

$$
\widehat{G}(s)=[G(s)]^{-T},
$$

where

$$
\widehat{G}(s)=\left[\begin{array}{ccc}
\frac{1}{\widehat{g}_{11}(s)} & \cdots & \frac{1}{\widehat{g}_{1 n}(s)} \\
\vdots & \ddots & \vdots \\
\frac{1}{\widehat{g}_{n 1}(s)} & \cdots & \frac{1}{\widehat{g}_{n n}(s)}
\end{array}\right] .
$$

Generally, the interactions in most industrial models [12] can be effectively represented by a FOPDT model of the following form:

$$
g(s)=\frac{k e^{-\theta s}}{\tau s+1},
$$

where $k, \theta$, and $\tau$ are the steady gain, the time delay, and the time constant, respectively.

If the controlled multivariable system is well paired, the control loop transfer functions will have similar frequency properties in both situations: the other loops are closed or open. Hence, the ETFs have the same structures (5) as the corresponding open-loop transfer functions with three different parameters, that is, $\widehat{k}, \widehat{\theta}$, and $\widehat{\tau}[22,23]$.

Applying (3), the ETF between the $i$ th manipulated variable and the $i$ th controlled variable can be derived as

$$
\frac{\widehat{\tau}_{i i} s+1}{\widehat{k}_{i i}} e^{\hat{\theta}_{i i} s}=\widehat{g}_{i i}=\frac{\operatorname{adj} G_{i i}}{|G|},
$$


where $|G|$ is the determinant of $G(s)$ and $\operatorname{adj} G_{i i}$ is the $i$ th row and the $i$ th column element of the adjoint matrix of $G(s)$.

Applying derivative with respect to $s$ on both sides of (6), it yields

$$
\begin{gathered}
\frac{\widehat{\tau}_{i i} e^{\widehat{\theta}_{i i} s}+\left(\widehat{\tau}_{i i} s+1\right) \widehat{\theta}_{i i} e^{\widehat{\theta}_{i i} s}}{\widehat{k}_{i i}} \\
=-\frac{1}{|G|^{2}} \sum_{p=1}^{n}\left(\sum_{q=1}^{n}\left(\operatorname{adj} G_{i q}\right) g_{q p}^{\prime}\right) \operatorname{adj} G_{p i},
\end{gathered}
$$

where $g_{q p}^{\prime}$ is the first derivative of $g_{q p}$.

Differentiating again both sides of (7), we have

$$
\begin{aligned}
& \frac{2 \widehat{\tau}_{i i} \widehat{\theta}_{i i} \hat{e}^{\widehat{\theta}_{i i} s}+\left(\tau_{i i} s+1\right) \widehat{\theta}_{i i}^{2} e^{\widehat{\theta}_{i i} s}}{\widehat{k}_{i i}}=-\frac{1}{|G|^{2}} \\
& \cdot \sum_{p=1}^{n}\left(\sum_{q=1}^{n}\left(\operatorname{adj} G_{i q}\right) g_{q p}^{\prime \prime}\right) \operatorname{adj} G_{p i}+\frac{2}{|G|^{3}} \\
& \cdot \sum_{m=1}^{n}\left(\sum_{l=1}^{n}\left(\sum_{p=1}^{n}\left(\sum_{q=1}^{n}\left(\operatorname{adj} G_{i q}\right) g_{q p}^{\prime}\right) \operatorname{adj} G_{p l}\right) g_{l m}^{\prime}\right) \operatorname{adj} G_{m i},
\end{aligned}
$$

where $g_{q p}^{\prime \prime}$ is the second derivative of $g_{q p}$.

Letting $s=0$, denoting $K=G(0)$

$$
|K|=\left|\begin{array}{ccc}
k_{11} & \cdots & k_{1 n} \\
\vdots & \ddots & \vdots \\
k_{n 1} & \cdots & k_{n n}
\end{array}\right|,
$$

and solving the equation system (6)-(8), the FOPDT model parameters of each ETF can be calculated as

$$
\begin{aligned}
& \hat{k}_{i i}=\frac{1}{a_{i i}}, \\
& \hat{\tau}_{i i}=\sqrt{\left(\frac{b_{i i}}{a_{i i}}\right)^{2}-\left(\frac{c_{i i}}{a_{i i}}\right),} \\
& \hat{\theta}_{i i}=\frac{b_{i i}}{a_{i i}}-\sqrt{\left(\frac{b_{i i}}{a_{i i}}\right)^{2}-\left(\frac{c_{i i}}{a_{i i}}\right)}=\frac{b_{i i}}{a_{i i}}-\tau_{i i},
\end{aligned}
$$

where

$$
\begin{aligned}
a_{i i} & =\frac{\operatorname{adj} K_{i i}}{|K|}, \\
b_{i i} & =-\frac{1}{|K|^{2}} \sum_{p=1}^{n}\left(\sum_{q=1}^{n}\left(\operatorname{adj} K_{i q}\right) g_{q p}^{\prime}(0)\right) \operatorname{adj} K_{p i}, \\
c_{i i} & =\frac{2}{|K|^{3}} \\
& \cdot \sum_{m=1}^{n} \sum_{l=1}^{n} \sum_{p=1}^{n} \sum_{q=1}^{n} \operatorname{adj} K_{i q} g_{q p}^{\prime}(0) \operatorname{adj} K_{p l} g_{l m}^{\prime}(0) \operatorname{adj} K_{m i} \\
& -\frac{1}{|K|^{2}} \sum_{p=1}^{n} \sum_{q=1}^{n} \operatorname{adj} K_{i q} g_{q p}^{\prime \prime}(0) \operatorname{adj} K_{p i} .
\end{aligned}
$$

$\operatorname{adj} K_{i q}, \operatorname{adj} K_{p i}, \operatorname{adj} K_{m i}$, and $\operatorname{adj} K_{p l}$ are simplified as the single formula using subscripts $v$ and $w$ as follows:

$$
\begin{aligned}
& \operatorname{adj} K_{v w}=(-1)^{v+w} \\
& \cdot\left|\begin{array}{cccccc}
k_{1,1} & \cdots & k_{1, v-1} & k_{1, v+1} & \cdots & k_{1, n} \\
\vdots & \ddots & \vdots & \vdots & \ddots & \vdots \\
k_{w-1,1} & \cdots & k_{w-1, v-1} & k_{w-1, v+1} & \cdots & k_{w-1, n} \\
k_{w+1,1} & \cdots & k_{w+1, v-1} & k_{w+1, v+1} & \cdots & k_{w+1, n} \\
\vdots & \ddots & \vdots & \vdots & \ddots & \vdots \\
k_{n, 1} & \cdots & k_{n, v-1} & k_{n, v+1} & \cdots & k_{n, n}
\end{array}\right|
\end{aligned}
$$

In order for the resulting FOPDT model to be feasible, $\widehat{\tau}_{i j}$ and $\widehat{\theta}_{i j}$ should be real and positive. It is clear from (10) that the following condition should be satisfied for finding feasible values:

$$
\left(\frac{b_{i i}}{a_{i i}}\right)^{2}>\frac{c_{i i}}{a_{i i}}>0 .
$$

Considering the interactions among control loops, we have decomposed the multivariable system into a set of SISO systems. In the following section, the stabilizing PID parametric region is determined from the equivalent open loops in formula (6) directly and independently.

\section{Stabilizing Parametric Regions}

This section provides a procedure to determine the complete set of stabilizing PID controllers for the equivalent single loop obtained in Section 2. The first step of this procedure is to determine the proportional gain range of the stabilizing PID controller. Then, for a fixed proportional gain within this range, the stabilizing integral and derivative parameter with known shape and boundaries are derived in the second step.

Consider the PID controller defined by

$$
g_{c}=k_{p}+\frac{k_{i}}{s}+k_{d} s
$$

where $k_{p}$ is the proportional gain, $k_{i}$ is the integral gain, and $k_{d}$ is the derivative gain.

Using the generalized theorem of Hermite-Biehler [9], the stabilizing range of proportional gain $k_{p}$ for the plant with transfer function shown in (6) is given by

$$
-\frac{1}{\widehat{k}}<k_{p}<\frac{1}{\widehat{k}}\left[\frac{\widehat{\tau}}{\hat{\theta}} \alpha_{1} \sin \left(\alpha_{1}\right)-\cos \left(\alpha_{1}\right)\right],
$$

where $\alpha_{1}$ is the solution of

$$
\tan (\alpha)=-\frac{\widehat{\tau}}{\widehat{\tau}+\widehat{\theta}} \alpha
$$

in the interval $\alpha \in(0, \pi)$. For $k_{p}$ values outside this range, there are no stabilizing $\mathbf{P}$ controllers.

For a fixed proportional gain calculated from formula (15), there are three conditions needed to be considered. 


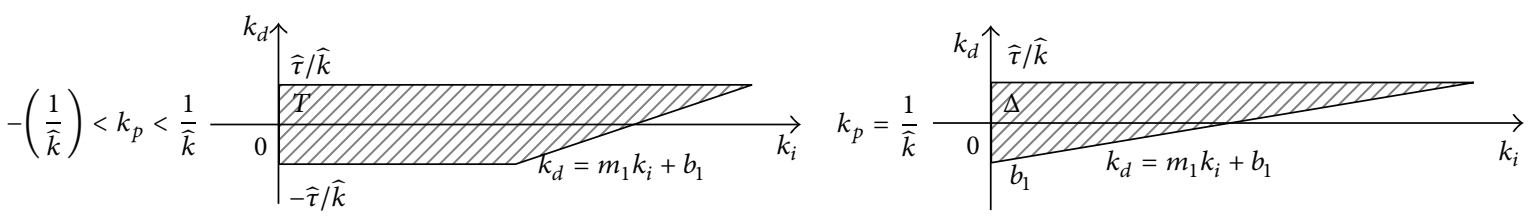

(a)

(b)

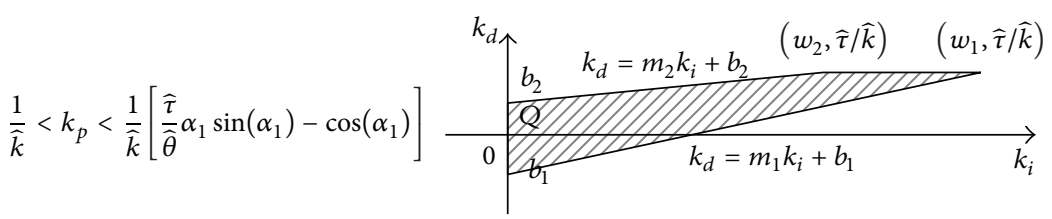

(c)

FIGURE 2: The stabilizing region of $\left(k_{i}, k_{d}\right)$.

The parameters $m_{j}, b_{j}$, and $\omega_{j}, j=1,2$, necessary for determining the stability boundaries of the areas $T, \Delta$, and $Q$ (as shown in Figure 2) can be calculated using (18)-(20), where $z_{j}(j=1,2)$ are the first and second positive-real solutions calculated from (17):

$$
\begin{aligned}
& \widehat{k} k_{p}+\cos (z)-\frac{\widehat{\tau}}{\widehat{\theta}} z \sin (z)=0, \\
& m_{j}=\frac{\widehat{\tau}^{2}}{z_{j}^{2}}, \\
& b_{j}=-\frac{\widehat{\tau}}{\widehat{k} z_{j}}\left[\sin \left(z_{j}\right)+\frac{\widehat{\tau}}{\widehat{\theta}} z_{j} \cos \left(z_{j}\right)\right], \\
& \omega_{j}=\frac{z_{j}}{\widehat{k} \widehat{\theta}}\left[\sin \left(z_{j}\right)+\frac{\widehat{\tau}}{\widehat{\theta}} z_{j}\left(\cos \left(z_{j}\right)+1\right)\right] .
\end{aligned}
$$

So far, a comprehensive method has been presented to compute the entire set of stabilizing PID controller parameters for a FOPDT model. By means of (15), the $k_{p}$-axis can be gridded. For each $k_{p}$, the stable region in the $\left(k_{d}, k_{i}\right)$-plane is determined by (17)-(20) and the stable region is a set of convex polygons.

In summary, the procedure for determining the stabilizing multiloop PID parameters is as follows.

Step 1. Calculate the ETF of the ith loop based on (10).

Step 2. Pick $k_{p}$ in the range determined by (15).

Step 3. Find the roots $z_{1}$ and $z_{2}$ of (17).

Step 4. Compute the parameters $m_{j}, b_{j}$, and $\omega_{j}, j=1,2$, associated with the previous solutions $z_{j}$ by using (18)-(20).

Step 5. According to the case in Figure 2, determine the stabilizing region in the $k_{i}-k_{d}$ space.

Step 6. Go to Step 1 until $i$ is equal to $n$.
The validity of the design is based on the validity of the SISO models (6). Thence, Assumption 1, (1b), should be checked. Its impact is analyzed in the example that follows.

Remark. In the previous development, a FOPDT model has been assumed, as shown in (5). This model has been considered as a good approximation for most industrial processes [12]. If a higher order model is assumed, the rationale is similar, but due to the larger number of parameters, the procedure becomes much more complicated.

\section{Simulation Study}

This example is presented to illustrate the procedure to determine all stabilizing PID parameters for multivariable system. It represents a pilot-scale distillation column consisting of an eight-tray plus reboiler separating methanol and water introduced by [24]

$$
G(s)=\left[\begin{array}{cc}
\frac{12.8 e^{-s}}{16.7 s+1} & \frac{-18.9 e^{-3 s}}{21 s+1} \\
\frac{6.6 e^{-7 s}}{10.9 s+1} & \frac{-19.4 e^{-3 s}}{14.4 s+1}
\end{array}\right] .
$$

Each ETF can be calculated by (10) as follows:

$$
\begin{aligned}
& \widehat{g}_{1}=\frac{6.37 e^{-0.31 s}}{10.53 s+1} \\
& \widehat{g}_{2}=\frac{-9.65 e^{-4.27 s}}{6.27 s+1}
\end{aligned}
$$

In terms of (15), the necessary conditions for the proportional controller to stabilize the multivariable system are $k_{p 1} \epsilon$ $(-0.16,9.85)$ and $k_{p 2} \in(-0.33,0.10)$. According to the algorithm in Section 4, stabilizing multiloop PID controller parameters are calculated and regions of stability for each loop are shown in Figures 3 and 4, respectively.

To demonstrate the effectiveness of the stabilizing regions drawn by Figures 3 and 4, two parameter sets of the PID controllers are selected as one is inside the stabilizing region and the other one is outside of the region. When $k_{p 1}=0.16 \epsilon$ $(-0.16,9.85)$ is selected in the first loop, the stabilizing region 


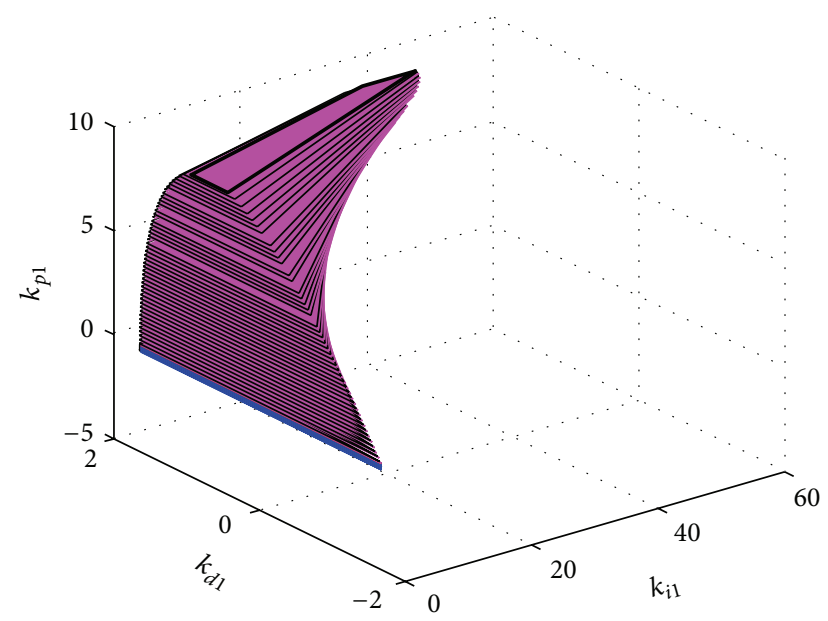

FIgURE 3: The stabilizing parametric region of loop 1.

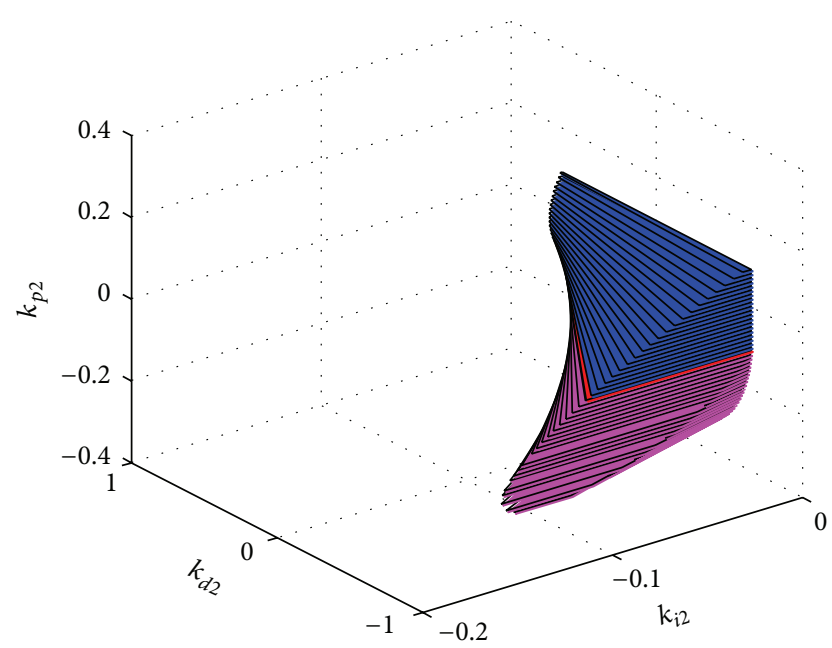

FIGURE 4: The stabilizing parametric region of loop 2.

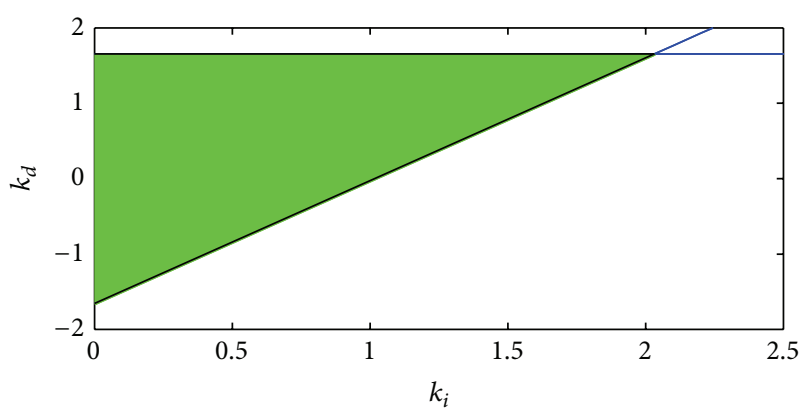

FIGURE 5: The stabilizing region of $\left(k_{i}, k_{d}\right)$ for $k_{p 1}=0.16$.

of $\left(k_{i}, k_{d}\right)$ is presented in Figure 5, which is determined by the parameters $z_{1}=0.241, m_{1}=1.628$, and $b_{1}=-1.6529$. The stable set $k_{p 1}=0.157, k_{i 1}=0.1$, and $k_{d 1}=1.0$ and unstable set $k_{p 1}=0.157, k_{i 1}=0.1$, and $k_{d 1}=1.66$ are chosen for loop 1 , respectively. The second loop selects the stabilizing PID parameters with $k_{p 1}=-0.1036, k_{i 1}=-0.002$, and $k_{d 1}=0$. Figure 6 shows the stable and the unstable responses of the
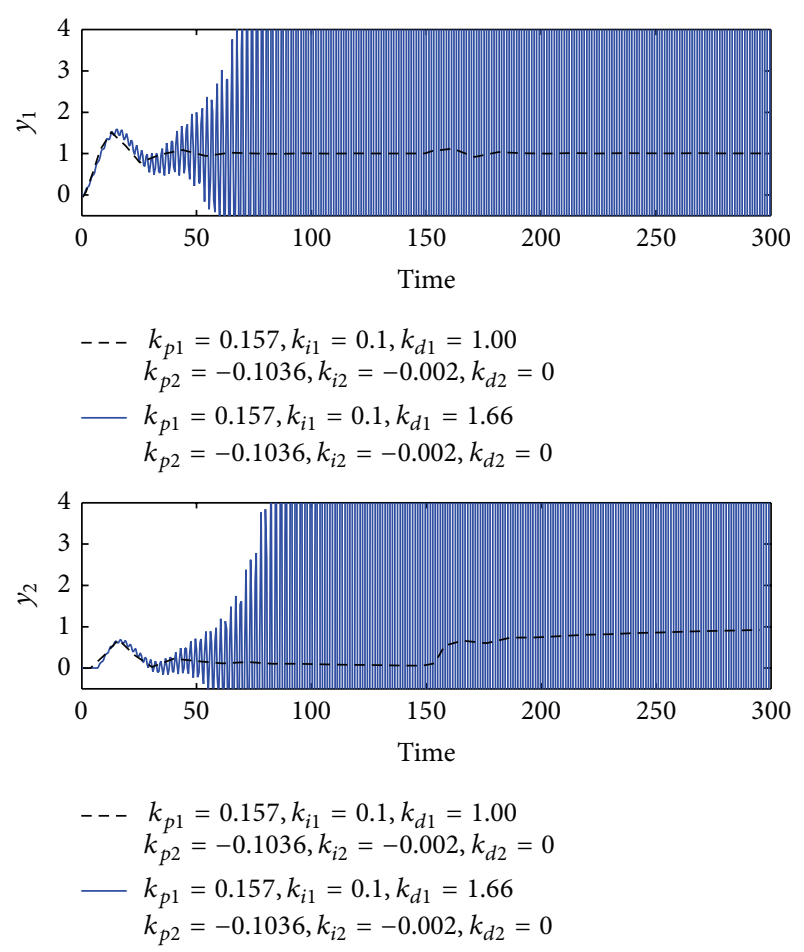

FIGURE 6: Step responses under stable and unstable controller parameters.

controlled process under set-point steps for each loop. The set-point changes are set in $t=0 \mathrm{~s}$ and $t=150 \mathrm{~s}$ for the first and second loop, respectively.

From Figure 6, we find that if the controller parameters of one loop surpass the stabilizing range, the whole controlled multivariable systems will be unstable.

Assumption Validation. For the set of stabilizing parameters selected in both loops, the Bode plots of the exact ETF in (1a) and the corresponding approximated ETF computed in (22) are depicted in Figure 7. Also the step responses of both ETFs (the exact one and the approximated) are plotted in Figure 8.

This illustrates the validity of the approximation and thence the whole procedure to design the PID parameters for the MIMO plant.

\section{Conclusions}

This paper presents a comprehensive method to compute the entire set of PID controller parameters for multivariable systems with the objective of closed-loop stabilization. The procedure to determine the complete set of stabilizing PID controllers is achieved in two stages. First, the MIMO system is transformed to a set of equivalent single loops, assuming perfect pairing control. Then, the range of proportional gains for equivalent single loops is calculated to determine the stabilizing regions in the space of integral and derivative gains. The results of this paper provide insight into designing and analyzing stabilizing PID for multivariable systems. Future work will be devoted to investigate the PID stabilizing ranges 

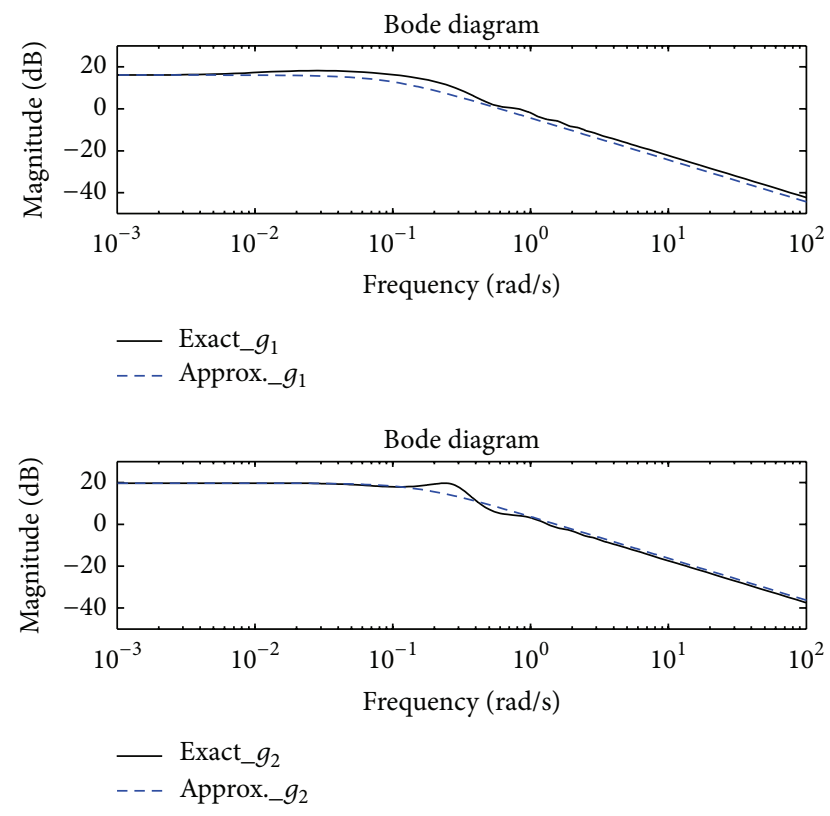

Figure 7: Bode plots of open-loop ETFs (exact and approx.).
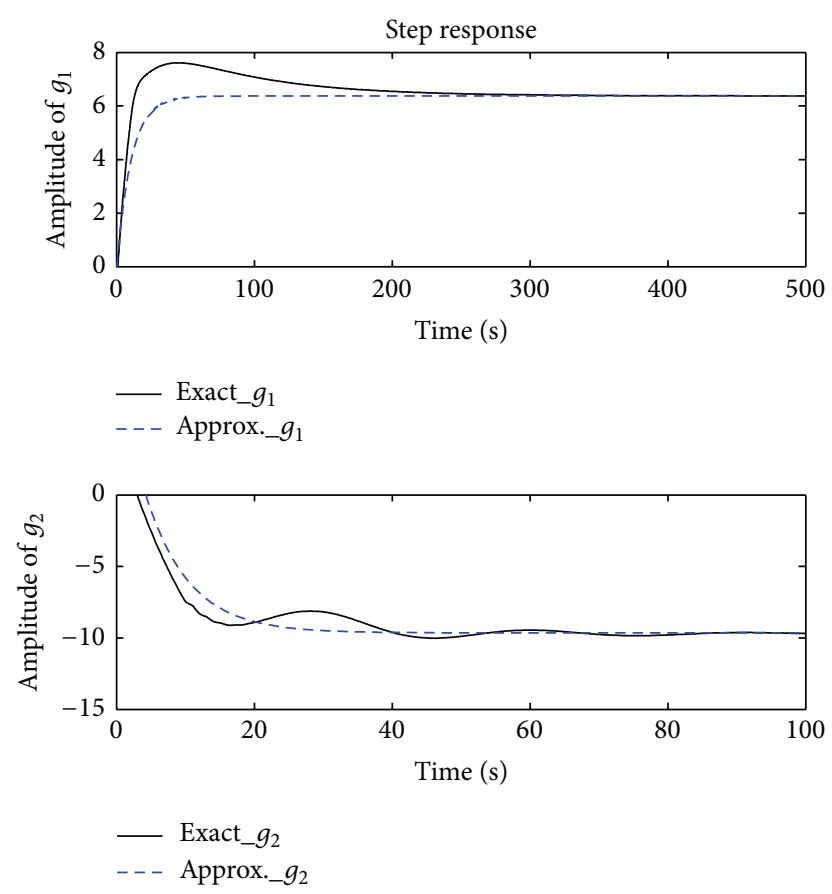

FIGURE 8: Step responses of open-loop ETFs (exact and approx.).

for linear multivariable systems under centralized control structure.

\section{Competing Interests}

The authors declare that they have no competing interests.

\section{References}

[1] Q.-G. Wang, Z. Ye, W.-J. Cai, and C.-C. Hang, PID Control for Multivariable Processes, vol. 373 of Lecture Notes in Control and Information Sciences, Springer, 2008.

[2] Z.-Y. Nie, Q.-G. Wang, M. Wu, and Y. He, "Tuning of multi-loop PI controllers based on gain and phase margin specifications," Journal of Process Control, vol. 21, no. 9, pp. 1287-1295, 2011.

[3] S. Gigi and A. K. Tangirala, "Quantification of interaction in multiloop control systems using directed spectral decomposition," Automatica, vol. 49, no. 5, pp. 1174-1183, 2013.

[4] T. N. L. Vu and M. Lee, "Independent design of multi-loop PI/PID controllers for interacting multivariable processes," Journal of Process Control, vol. 20, no. 8, pp. 922-933, 2010.

[5] H.-P. Huang, J.-C. Jeng, C.-H. Chiang, and W. Pan, "A direct method for multi-loop PI/PID controller design," Journal of Process Control, vol. 13, no. 8, pp. 769-786, 2003.

[6] M.-J. He, W.-J. Cai, B.-F. Wu, and M. He, "Simple Decentralized PID controller design method based on dynamic relative interaction analysis," Industrial \& Engineering Chemistry Research, vol. 44, no. 22, pp. 8334-8344, 2005.

[7] Q.-G. Wang, C. Lin, Z. Ye, G. Wen, Y. He, and C. C. Hang, "A quasi-LMI approach to computing stabilizing parameter ranges of multi-loop PID controllers," Journal of Process Control, vol. 17, no. 1, pp. 59-72, 2007.

[8] G. J. Silva, A. Datta, and S. P. Bhattacharyya, "PI stabilization of first-order systems with time delay," Automatica, vol. 37, no. 12, pp. 2025-2031, 2001.

[9] G. J. Silva, A. Datta, and S. P. Bhattacharyya, "New results on the synthesis of PID controllers," IEEE Transactions on Automatic Control, vol. 47, no. 2, pp. 241-252, 2002.

[10] H. Xu, A. Datta, and S. P. Bhattacharyya, "PID stabilization of LTI plants with time delay," in Proceedings of 42nd IEEE International Conference on Decision and Control, pp. 40384043, Maui, Hawaii, USA, December 2003.

[11] M.-T. Ho, G. J. Suva, A. Datta, and S. P. Bhattacharyya, "Real and complex stabilization: Stability and performance," in Proceedings of the American Control Conference (AAC '04), pp. 4126-4138, Boston, Mass, USA, July 2004.

[12] M. T. Ho, G. J. Silva, A. Datta, and S. P. Bhattacharyya, PID Controllers for Time Delay Systems, Birkhauser, Boston, Mass, USA, 2005.

[13] G. J. Silva, A. Datta, and S. P. Bhattacharyya, "Robust control design using the PID controller," in Proceedings of 41st IEEE on International Conference on Decision and Control, pp. 1313-1318, Las Vegas, Nev, USA, December 2002.

[14] K. S. Kim, Y. C. Kim, L. H. Keel, and S. P. Bhattacharyya, "PID controller design with time response specifications," in Proceedings of the American Control Conference, pp. 5005-5010, June 2003.

[15] L. H. Keel and S. P. Bhattacharyya, "Controller synthesis free of analytical models: three term controllers," IEEE Transactions on Automatic Control, vol. 53, no. 6, pp. 1353-1369, 2008.

[16] L.-1. Ou, W.-D. Zhang, and L. Yu, "Low-order stabilization of LTI systems with time delay," IEEE Transactions on Automatic Control, vol. 54, no. 4, pp. 774-787, 2009.

[17] B. Wang, D. Rees, and Q.-C. Zhong, "Control of integral processes with dead time. Part IV: various issues about PI controllers," IEE Proceedings-Control Theory and Applications, vol. 153, no. 3, pp. 302-306, 2006. 
[18] L. Ou, Y. Tang, D. Gu, and W. Zhang, "Stability analysis of PID controllers for integral processes with time delay," in Proceedings of the American Control Conference (ACC '05), pp. 4247-4252, June 2005.

[19] L. Ou, W. Zhang, and D. Gu, "Sets of stabilising PID controllers for second-order integrating processes with time delay," IEE Proceedings: Control Theory and Applications, vol. 153, no. 5, pp. 607-614, 2006.

[20] C. Rajapandiyan and M. Chidambaram, "Controller design for MIMO processes based on simple decoupled equivalent transfer functions and simplified decoupler," Industrial and Engineering Chemistry Research, vol. 51, no. 38, pp. 12398-12410, 2012.

[21] Y. Shen, W.-J. Cai, and S. Li, "Normalized decoupling control for high-dimensional MIMO processes for application in room temperature control HVAC systems," Control Engineering Practice, vol. 18, no. 6, pp. 652-664, 2010.

[22] Q. Xiong and W.-J. Cai, "Effective transfer function method for decentralized control system design of multi-input multioutput processes," Journal of Process Control, vol. 16, no. 8, pp. 773-784, 2006.

[23] I.-L. Chien, H.-P. Huang, and J.-C. Yang, "A simple multiloop tuning method for PID controllers with no proportional kick," Industrial and Engineering Chemistry Research, vol. 38, no. 4, pp. 1456-1468, 1999.

[24] R. K. Wood and M. W. Berry, "Terminal composition control of a binary distillation column," Chemical Engineering Science, vol. 28, no. 9, pp. 1707-1717, 1973. 


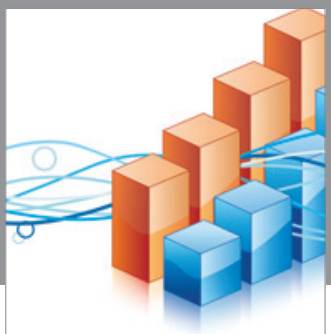

Advances in

Operations Research

vatem alat4

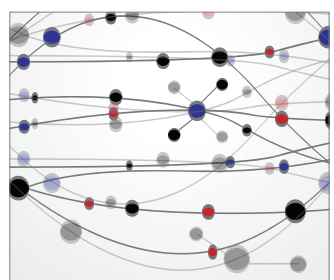

\section{The Scientific} World Journal
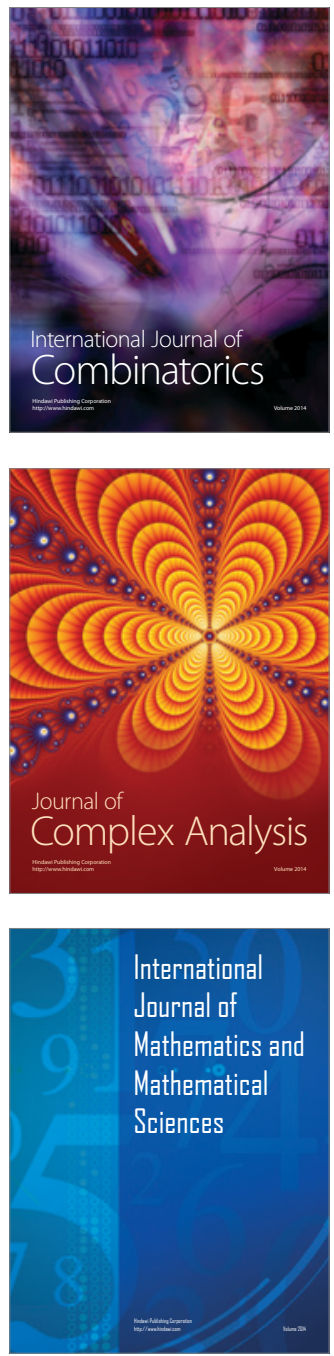
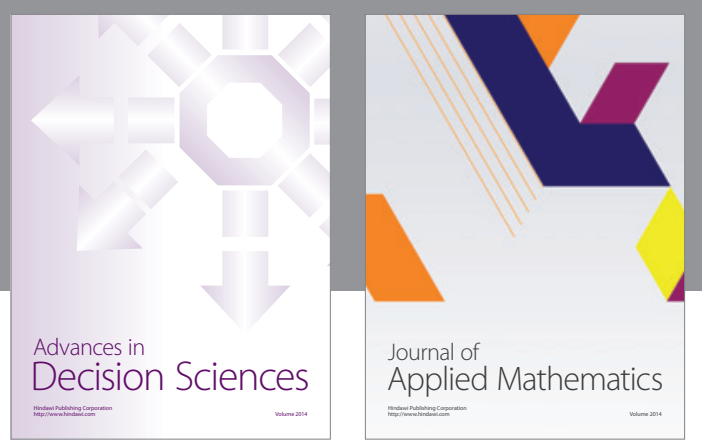

Algebra

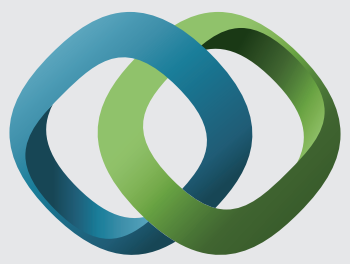

\section{Hindawi}

Submit your manuscripts at

http://www.hindawi.com
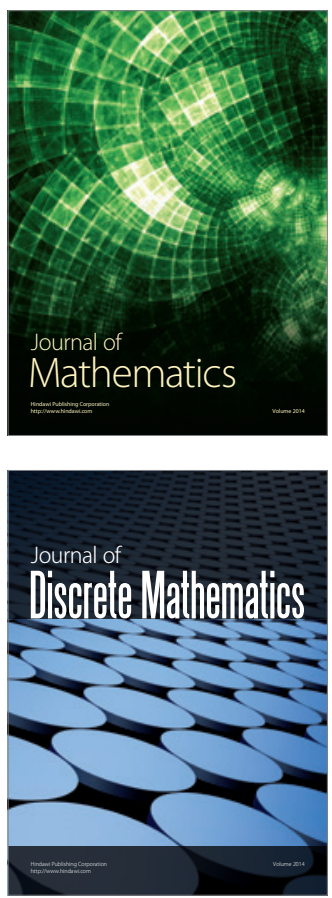

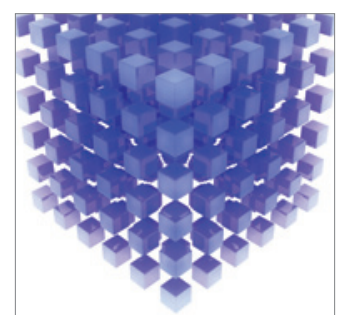

Mathematical Problems in Engineering
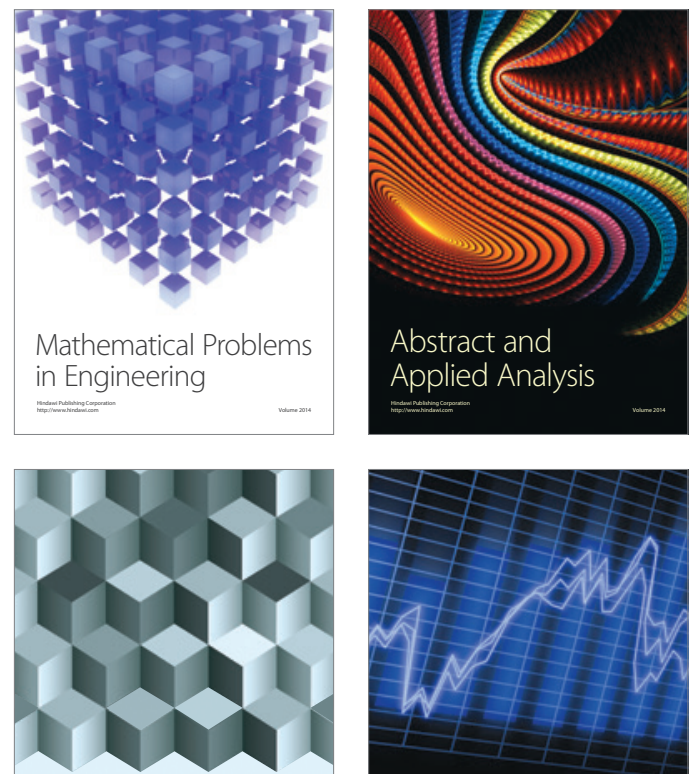

Journal of

Function Spaces

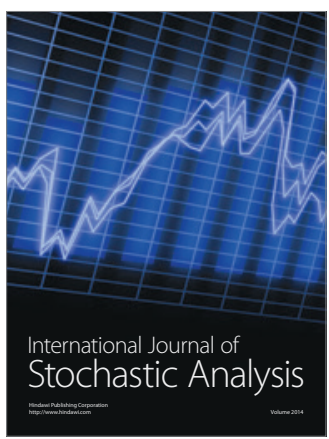

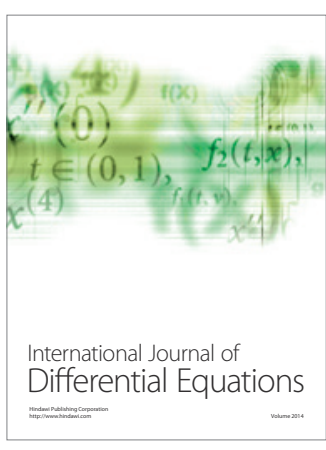
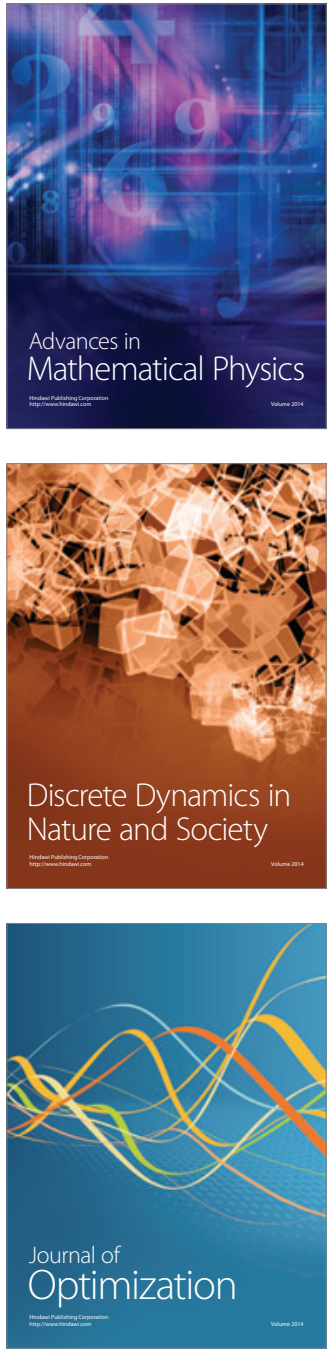